\title{
ON HYPERSURFACES OF HYPERBOLIC SPACE INFINITESIMALLY SUPPORTED BY HOROSPHERES
}

\author{
ROBERT J. CURRIER
}

\begin{abstract}
This paper is concerned with complete, smooth immersed hypersurfaces of hyperbolic space that are infinitesimally supported by horospheres. This latter condition may be restated as requiring that all eigenvalues of the second fundamental form, with respect to a particular unit normal field, be at least one. The following alternative must hold: either there is a point where all the eigenvalues of the second fundamental form are strictly greater than one, in which case the hypersurface is compact, imbedded and diffeomorphic to a sphere; or, the second fundamental form at every point has 1 as an eigenvalue, in which case the hypersurface is isometric to Euclidean space and is imbedded in hyperbolic space as a horosphere.
\end{abstract}

This paper is devoted to the consideration of complete, immersed, $C^{\infty}$ hypersurfaces of hyperbolic space that are infinitesimally supported by horospheres. By the latter phrase, we mean that, with respect to the appropriately chosen normal, the second fundamental form is greater than or equal to the identity, the second fundamental form of a horosphere. Equivalently, all the eigenvalues of the second fundamental form of the hypersurface are greater than or equal to 1 . The results are given in the following theorems:

Theorem A. Suppose $M$ is a complete, connected $C^{\infty}$ Riemannian manifold whose dimension, $n$, is at least 2. Suppose that $f: M \rightarrow H^{n+1}$ is a $C^{\infty}$ isometric immersion and that there exists a smooth normal field $\nu$ along $f$ such that, at every point of $M$, all the eigenvalues of the second fundamental form of $M$ in $H^{n+1}$ with respect to $\nu$ are at least 1 . If there is a point $p$ in $M$ where all the eigenvalues of $\mathrm{II}_{p}$ are strictly greater than 1 , then (1) $M$ is compact, (2) $M$ is imbedded as the boundary of a convex body, and (3) $M$ is diffeomorphic to $S^{n}$.

Theorem B. Suppose that $M, f$ and $\nu$ are as in Theorem A. If the least eigenvalue of II at every point is 1 , then (1) $M$ is isometric to $R^{n}$, and (2) $M$ is imbeded as a horosphere.

The Gauss equation implies that such a hypersurface has nonnegative intrinsic sectional curvature. Thus, Theorems A and B form a partial analogue of

Received by the editors January 301987 and, in revised form, January 5, 1988.

1980 Mathematics Subject Classification (1985 Revision). Primary 53C42; Secondary 53B25, $52 \mathrm{~A} 55$. 
Sacksteder's Theorem [10] in Euclidean space. Indeed, the proof of Theorem $B$ is inspired by the techniques of Sacksteder's proof. If the hypersurface were assumed compact, then do Carmo and Warner's theorem [5] would already give the result from the weaker hypothesis that the second fundamental form is semidefinite.

In the context of hypersurfaces of arbitrary Hadamard manifolds (complete, simply-connected manifolds of nonpositive curvature), of which hyperbolic space is an example, Alexander [1] obtained a result by assuming that the eigenvalues of the second fundamental form have appropriately bounded absolute values. The special case of this theorem in hyperbolic space states that if the absolute values of the eigenvalues of the second fundamental form are bounded from above by 1 , then the hypersurface is imbedded and diffeomorphic to $R^{n}$. Thus Theorem B can be thought of as a "rigidity theorem" between Theorem A and Alexander's Theorem.

A different approach in this setting was used by Bolton [2], who obtained a similar imbedding result from the hypothesis that tangent horospheres are globally supporting. In the proof of Theorem A, we shall see that tangent horospheres are globally supporting, but the proof of imbeddedness given here does not depend on this result.

Rather, the proof of Theorem A is inspired by do Carmo and Lima's proof [4] of a special case of Sacksteder's result in $R^{n+1}$. They assume that the second fundamental form at some point is definite; later, de Miranda Gomes [3] combined this result with a result of Jonker [7] to give a proof of Sacksteder's theorem in its full generality. However, there appears to be a small gap in the published version of do Carmo and Lima's proof, which may be overcome as indicated in $\S 3$ if the dimension of the hypersurface is at least 3 . It seens necessary to consider that the only entirely correct proof for the case of surfaces in $R^{3}$ is the one due to Sacksteder.

$\S 2$ contains introductory materials, and concludes with a proof that tangent horospheres locally support the hypersurface. $\S 3$ contains a proof of Theorem $A$ and a discussion of the Euclidean situation as treated by do Carmo and Lima; and $\S 4$ contains the proof of Theorem $B$.

I would like to thank my advisor, Professor Stephanie Alexander, for her criticism and encouragement during the course of this work. I would also like to thank Professors Felix Albrecht, I. David Berg, Richard L. Bishop and George K. Francis for many stimulating conversations. A portion of this paper constitutes part of the author's Dissertation at the University of Illinois at Urbana-Champaign.

\section{HYPERBOLIC SPACE}

To simplify computations we shall often use the upper half-space model of $H^{n+1}$. In this model, $H^{n+1}$ is represented as the domain $\left\{\left(x_{0}, x_{1}, \ldots, x_{n}\right) \in\right.$ $\left.R^{n+1}: x_{0}>0\right\}$ with the metric $g=\left(1 / x_{0}^{2}\right) \sum_{i=0}^{n} d x_{i}^{2}$. To further simplify the 
calculations, it is convenient to use the global orthonormal frame field given by $X_{i}=x_{o} \partial_{i}$ rather than the coordinate fields $\partial_{i}$ alone. These vector fields satisfy the relations:

$$
\begin{array}{ll}
\nabla_{X_{0}} X_{i}=0 & \text { for all } i, \\
\nabla_{X_{i}} X_{0}=-X_{i} & \text { for } i>0, \\
\nabla_{X_{i}} X_{j}=\delta_{i j} X_{0} & \text { for } i, j>0 .
\end{array}
$$

In particular, these relations indicate that the frame is parallel along lines in the model parallel to the $x_{0}$-axis, which are geodesics. In brief, we shall refer to this direction as "vertical". The "horizontal" hypersurfaces defined by the equation $x_{0}=$ constant are horospheres. They are totally umbilic hypersurfaces whose second fundamental form with respect to the normal $-X_{0}$ is the identity matrix.

If $P$ is a horosphere, then the horoball bounded by $P$ is the open component of the complement of $P$ in $H^{n+1}$ that is a convex set. In the case that $P$ is defined by the equation $x_{0}=c$, the horoball bounded by $P$ is defined by the inequality $x_{0}>c$.

If $M$ is a hypersurface in $H^{n+1}$, and $\nu$ is a local unit normal vector field near $p$ in $M$, then the second fundamental form of $M$ at $p$ is given by $\mathrm{II}_{p}\left(X_{p}, Y_{p}\right)=\left\langle\nabla_{X} \nu, Y\right\rangle_{p}$ for vector fields $X$ and $Y$ tangent to $M$. If all the eigenvalues of II $_{p}$ are greater than or equal to 1 , we say that $M$ is infinitesimally supported by a horosphere at $p$.

Lemma 1. Suppose $M$ is a smooth hypersurface of $H^{n+1}$ and $U$ is an open set in $M$ on which there is a smooth normal vector field $\nu$ such that every point of $U$ is infinitesimally supported by a horosphere. Then every point of $U$ is locally supported by a horosphere.

Proof. Let $p$ be a point of $U$. Choose upper half-space coordinates so that $p$ is $(1,0, \ldots, 0)$ and $\nu_{p}=-X_{0}$. Then $p$ has a neighborhood $V$ contained in $U$ that can be described as the graph of a function $x_{0}=h(\bar{x})$ for $\bar{x}$ in some domain in $R^{n}$. The isometries of $H^{n}$ that fix $\nu_{p}$ are the rotations of the upper half-space model that leave the $x_{0}$-axis pointwise fixed. Therefore they do not alter the (Euclidean) properties of the model, in particular, convexity of the graph. Thus, it suffices to consider the behavior of $h$ along the $x_{1}$-axis. Define a vector field tangent to $V$ by $X_{1}+\left(\partial h / \partial x_{1}\right) X_{0}$, and denote it by $W$. The normal field $\nu$ on $V$ can be written as

$$
\nu=\left(1+\sum_{i=1}^{n}\left(\frac{\partial h}{\partial x_{i}}\right)^{2}\right)^{-1 / 2}\left(-X_{0}+\sum_{i=1}^{n}\left(\frac{\partial h}{\partial x_{i}}\right) X_{i}\right) .
$$

Then, by the definition of $W$ and the formulas for $\nabla$,

$$
\mathrm{II}(W, W)=\left(1+\sum_{i=1}^{n}\left(\frac{\partial h}{\partial x_{i}}\right)^{2}\right)^{-1 / 2}\left(1+h \frac{\partial^{2} h}{\partial x_{1}^{2}}+\left(\frac{\partial h}{\partial x_{1}}\right)^{2}\right) .
$$


Since, by assumption, $\operatorname{II}(W, W) \geq|W|^{2}$ then

$$
1+h \frac{\partial^{2} h}{\partial x_{1}^{2}}+\left(\frac{\partial h}{\partial x_{1}}\right)^{2} \geq 1+\left(\frac{\partial h}{\partial x_{1}}\right)^{2} .
$$

Therefore $h$ is a convex function and $V$ is supported by its Euclidean tangent plane at $p$, namely the horosphere $\left\{x_{0}=1\right\}$.

\section{The proof of Theorem A}

This section will be concerned with the proof of:

Theorem A. Suppose $M$ is a complete, connected $C^{\infty}$ Riemannian manifold whose dimension, $n$, is at least 2. Suppose that $f: M \rightarrow H^{n+1}$ is a $C^{\infty}$ isometric immersion and that there exists a smooth normal field $\nu$ along $f$ such that, at every point of $M$, all the eigenvalues of the second fundamental form of $M$ in $H^{n+1}$ with respect to $\nu$ are at least one. If there is a point $p$ in $M$ where all the eigenvalues of II $_{p}$ are strictly greater than 1 , then (1) $M$ is compact, (2) $M$ is imbedded as the boundary of a convex body, and (3) $M$ is diffeomorphic to $S^{n}$.

Proof. Fix upper half-space coordinates on $H^{n+1}$ so that $f(p)=(1,0, \ldots, 0)$ and $\nu_{p}=-X_{0}$. Define a height function $h: M \rightarrow \mathbf{R}$ by the equation $h(q)=$ $x_{0}(f(q))-1$. The proof consists of showing that $h$ is a Morse funtion on $M$ with exactly two critical points and that $f$ imbeds the intermediate level sets as the boundary of convex sets in the horospheres $\left\{x_{0}=\right.$ constant $\}$.

Since $\nu_{p}=-X_{0}$, the Euclidean gradient of $h$ at $p$ vanishes, so a computation like Lemma 1 expresses the Hessian, $\nabla^{2} h(p)$, by the equation $\nabla^{2} h(p)=$ $\mathrm{II}_{p}-$ Id. Since all the eigenvalues of $\mathrm{II}_{p}$ are strictly greater than one, $\nabla^{2} h(p)$ is positive definite, and $p$ is a strict local minimum of $h$. Further, the components of level sets of $h$ near $p$, when nonempty, are diffeomorphic to spheres. Following do Carmo-Lima, we shall call a component of the level set of $h$ at level $s$ a normal component if the following conditions are satisfied:

(1) The component, which we denote $M_{s}$, is diffeomorphic to $S^{n-1}$ and bounds an open region in $M$, which we denote $D_{s}$, that contains exactly one critical point of $h$, namely $p$.

(2) There exists a homeomorphism from the closed ball $\mathrm{Cl}[B(s)]$ in $\mathbf{R}^{n}$ onto $\mathrm{Cl}\left[D_{s}\right]$ such that the image of each sphere $S(t)$ is the normal component at levet $t$, for all $t$ in the interval $(0, s)$.

If these conditions are satisfied we shall also call $D_{s}$ a normal region and $s$ a normal value. From the preceding discussion, we see that there do exist positive normal values. The proof continues by following the normal component $M_{s}$ as $s$ increases. Let $D^{*}$ denote the union of all normal regions, and let $s^{*}$ denote the supremum of all normal values. Then $D^{*}$ is nonempty and is open in $M$. One of the following mutually exclusive cases must occur.

(i) $D^{*}=M$; 
(ii) $D^{*} \neq M$ and the topological boundary of $D^{*}$, which we shall denote $M^{*}$, contains a critical point of $h$; or

(iii) $D^{*} \neq M$ and $M^{*}$ contains no critical point of $h$.

The theorem will be proved by showing, first, that case (iii) is impossible, second, that in case (ii) the critical point in $M^{*}$ is a nondegenerate maximum, from which the conclusion of the theorem follow, and third, that case (i) is also impossible.

Let $P_{s}$ denote the horosphere $\left\{x_{0}=s+1\right\}$, which contains $f\left(M_{s}\right)$. Let $f_{s}$ : $M_{s} \rightarrow P_{s}$ denote the immersion of the normal component in its corresponding horosphere. Since $M_{s}$ contains no critical point of $h$, it is possible to choose a unit normal field $\nu_{s}$ along $f_{s}$ with the property that $\left\langle\nu, \nu_{s}\right\rangle>0$. [That is, $\nu_{s}$ is perpendicular to $f_{s}\left(M_{s}\right)$ in $P_{s}$ and points toward the same side of $f_{s}\left(M_{s}\right)$ as the projection of $\nu$ into $P_{s}$.] Let $\mathrm{II}_{s}$ be the second fundamental form of $M_{s}$ in $P_{s}$ with respect to this normal. For any $s, 0<s<s^{*}$, the form $\mathrm{II}_{s}$ at a point $q$ can be seen to be positive definite, because, by Lemma $1, M$ is locally supported by a horosphere near $q$, and the intersection of this horosphere with $P_{s}$ is a sphere and so strictly convex.

For $s$ in the interval $\left(0, s^{*}\right)$, we know that $M_{s}$ is compact and $\mathrm{II}_{s}$ is positive definite. Consequently, $M_{s}$ is embedded in $P_{s}$ as a hypersurface that is diffeomorphic to $S^{n}$ and that bounds a convex body. In the case that $n>2$, this result follows immediately from a theorem of Hadamard [6], since $P_{s}$ is isometric to $\mathbf{R}^{n}$. In the case that $n=2$, then $M_{s}$ is a curve in $P_{s}$, which is isometric to $\mathbf{R}^{2}$. The preceding paragraph shows that the curvature of this curve does not change sign, so it bounds a convex body if and only if it is imbedded. For small positive $s$, we know that $M_{s}$ is imbedded, since the critical point $p$ is nondegenerate. Since $M_{s}$ is compact for each $s$ in the interval $\left(0, s^{*}\right)$, the normal map $\nu_{s}: M_{s} \rightarrow S^{1}$ is onto. The rotation of this map is +1 for small positive $s$ and is continuous, since there are no critical points of $h$ other than $p$ in $D^{*}$. Consequently, the rotation of $\nu_{s}$ is +1 for all $s$ in $\left(0, s^{*}\right)$; therefore $M_{s}$ is imbedded and bounds a convex body in $P_{s}$.

A major step will be to show that case (iii) is impossible; that is, it is impossible to have $D^{*} \neq M$ with no critical point of $h$ in $M^{*}$, the boundary of $D^{*}$. If this were possible, we shall see that $M^{*}$ would be a noncompact, complete, convex hypersurface of $P_{s}$.

Arguments analogous to do Carmo-Lima [4] show that every point $q$ of $M^{*}$ has $h(q)=s^{*}$, that every connected component of the level set $\left\{h=s^{*}\right\}$ that contains a point of $M^{*}$ is entirely contained in $M^{*}$, that $M^{*}$ is noncompact, and that $M^{*}$ bounds a convex body in $P_{s^{*}}$. In order that the exposition here be as complete as possible, indications of these results follow.

If a point $q$ is in $M^{*}$, certainly $h(q) \leq s^{*}$, since on $D^{*}$ we have the inequaltiy $h<s^{*}$. If $h(q)=s^{\prime}$ with $s^{\prime}<s^{*}$, then $M_{s^{\prime}}$ is a normal component not containing any critical point of $h$, so it has a neighborhood in $D^{*}$ composed 
of points with levels close to $s^{\prime}$. Since $h$ is continuous, $M_{s^{\prime}}$ passes through $q$ and $q$ is not in $M^{*}$.

To see that the connected component $C$ of the level set $\left\{h=s^{*}\right\}$ that contains a point of $M^{*}$ is entirely contained in $M^{*}$, use the trajectories of $\operatorname{grad} h$ to follow the normal components $M_{s}$ as $s$ approaches $s^{*}$. Let $A$ be the intersection of $C$ with $M^{*}$. Then $A$ is closed in $C$, since $M^{*}$ is closed in $M$. But $A$ is also open in $C$, as can be verified by following the trajectories of $\operatorname{grad} h$. Since $C$ is connected, $A$ must equal $C$; equivalently, $C$ is contained in $M^{*}$. Consequently, $M^{*}$ is complete, since it is a closed submanifold of $M$, which is complete.

If $M^{*}$ were compact, then it would be diffeomorphic to $S^{n-1}$, like the normal levels $M_{s}$. Since, in case (iii), $M^{*}$ contains no critical point of $h$, the flow of $\operatorname{grad} h$ would give a normal level higher than $s^{*}$. This would contradict the definition of $s^{*}$.

If $M^{*}$ did not bound a convex body in $P_{s^{*}}$, then there would be a point $q$ in $M^{*}$ for which a pair of points $q_{1}$ and $q_{2}$ of $M^{*}$ lie on opposite sides of the tangent space in $P_{s^{*}}$ of $M^{*}$ at $q$. Let $\varphi, \varphi_{1}$ and $\varphi_{2}$ be the trajectories of $\operatorname{grad} h$, parametrized by $s$, which pass through, respectively, $q, q_{1}$ and $q_{2}$ when $s=s^{*}$. Then, for $s$ sufficiently close to $s^{*}$, the points $\varphi_{1}(s)$ and $\varphi_{2}(s)$ lie on opposite sides of the tangent space in $P_{s}$ of $M_{s}$ at $\varphi(s)$. This would contradict the convexity of $M_{s}$ in $P_{s}$.

It remains to show that $M^{*}$ is bounded, which contradiction eliminates case (iii) as a possibility. Specifically, it will be shown that the projection along vertical geodesics to the horosphere $P_{s^{*}}$ takes $\mathrm{Cl}\left[D^{*}\right]$ to a bounded set. This demonstration proceeds by following projected trajectories of $\operatorname{grad} h$ until they reach the boundary of the projection.

Fix a small positive number $s_{0}$ such that, at every point of $\mathrm{Cl}\left[D_{s_{0}}\right]$, the normal $\nu$ satisfies $\left\langle\nu, X_{0}\right\rangle<0$. There are no critical points of $h$ in the set $\mathrm{Cl}\left[D^{*}\right]-D_{s_{0}}$, which we shall denote $C$. Through each point of $C$ there is a unique trajectory of $\operatorname{grad} h$, and each trajectory contains a point of $M_{s_{0}}$. Reparametrize these trajectories by arclength $\tau$ from their points in $M_{s_{0}}$. Along each trajectory, $\varphi(\tau)$, define functions $\alpha(\tau)$ and $\beta(\tau)$ for which $\varphi^{\prime}(\tau)=$ $\alpha(\tau) X_{0}+\beta(\tau) \nu_{s(\tau)}$. These functions satisfy $\alpha(\tau)>0$, since $\varphi$ is a reparametrized trajectory of $\operatorname{grad} h$, and $\alpha(\tau)^{2}+\beta(\tau)^{2}=1$. Further, the sign of $\beta$ is determined by the sign of $\left\langle\nu,-X_{0}\right\rangle$. Recall that $\nu_{s(\tau)}$ is the normal in $P_{s(\tau)}$ to $M_{s(\tau)}$. Then

$$
\begin{aligned}
\mathrm{II}\left(\varphi^{\prime}, \varphi^{\prime}\right) & =-\left\langle\nu, \nabla_{\varphi^{\prime}} \varphi^{\prime}\right\rangle \\
& =-\left\langle\nu, \nabla_{\varphi^{\prime}}\left[\alpha(\tau) X_{0}+\beta(\tau) \nu_{s(\tau)}\right]\right\rangle \\
& =-\left\langle\nu, \alpha^{\prime}(\tau) X_{0}+\beta^{\prime}(\tau) \nu_{s(\tau)}-\alpha(\tau) \beta(\tau) \nu_{s(\tau)}+\beta(\tau)^{2}\left[X_{0}+\nabla_{\nu_{s}}^{P} \nu_{s}\right]\right\rangle .
\end{aligned}
$$

The last term is perpendicular to $\nu$, and $\nu=-\beta(\tau) X_{0}+\alpha(\tau) \nu_{s}$, so

$$
\mathrm{II}\left(\varphi^{\prime}, \varphi^{\prime}\right)=\alpha^{\prime}(\tau) \beta(\tau)-\beta^{\prime}(\tau) \alpha(\tau)+\beta(t)\left[\alpha(\tau)^{2}+\beta(\tau)^{2}\right] .
$$


Since $\alpha(\tau)^{2}+\beta(\tau)^{2}=1$, then $\alpha(\tau) \alpha^{\prime}(\tau)+\beta(\tau) \beta^{\prime}(\tau)=0$. Therefore

$$
\mathrm{II}\left(\varphi^{\prime}, \varphi^{\prime}\right)=-\left[\beta^{\prime}(\tau) / \alpha(\tau)\right]+\beta(\tau) \text {. }
$$

The hypothesis that all the eigenvalues of II are at least one implies that $\operatorname{II}\left(\varphi^{\prime}, \varphi^{\prime}\right)$ $\geq 1$, or equivalently, $\beta^{\prime}(\tau) \leq \alpha(\tau)[\beta(\tau)-1]<0$. We remark that the choice of $s_{0}$ implies that $\beta$ is positive everywhere on $M_{s_{0}}$.

Let $\pi: H^{n+1} \rightarrow P_{s^{*}}$ be the projection along vertical geodesics and $\eta$ the composite $\pi \circ f$. Then $\eta\left(M_{s}\right)$ is an imbedded, compact, convex hypersurface of $P_{s^{*}}$ for $s$ in the interval $\left(0, s^{*}\right)$. Define $A_{s}$ to be $\eta\left(\mathrm{Cl}\left[D_{s}\right]\right)$. Since $f\left(D_{s}\right)$ separates the exterior of the horosphere $P_{s}$, we can find a point of $f\left(D_{s}\right)$ that projects onto any interior point $q$ of the convex body in $P_{s^{*}}$ bounded by $\eta\left(M_{s}\right)$ by following the vertical geodesic through $q$ downwards. Thus any such point is contained in $A_{s}$. Since $D_{s}=\bigcup_{0 \leq t<s} M_{t}$, we have that $A_{t} \subset A_{s}$, if $0<t<s<s^{*}$. For $s$ in $\left(0, s^{*}\right), \mathrm{Cl}\left[D_{s}\right]$ is compact, hence $A_{s}$ is also.

Fix $s$ in the interval $\left(s_{0}, s^{*}\right)$. Let $q$ be a boundary point of $A_{s}$ in $P_{s^{*}}$. Then $q$ is $\eta(r)$ for some point $r$ in $\mathrm{Cl}\left[D_{s}\right]$. In fact, $r$ is not in $D_{s_{0}}$, since every point of $D_{s_{0}}$ projects to an interior point of $A_{s_{0}}$, because the function $\beta$ is positive for every trajectory as it crosses $M_{s_{0}}$. Let $\varphi:\left[\tau_{0}, \tau_{1}\right] \rightarrow M$ be the trajectory through $r$, parametrized by arclength, with $\varphi\left(\tau_{0}\right)$ in $M_{s_{0}}$ and $\varphi\left(\tau_{1}\right)=r$. We shall prove that $\beta\left(\tau_{1}\right) \geq 0$.

Suppose, on the contrary, that $\beta\left(\tau_{1}\right)<0$. Recall that $\varphi^{\prime}(\tau)=\alpha(\tau) X_{0}+$ $\beta(\tau) \nu_{s(\tau)}$, so $(\eta \circ \varphi)^{\prime}(\tau)=\pi_{*}\left(\beta(\tau) \nu_{s(\tau)}\right)$. We may interpret the sign of $\beta(\tau)$ as indicating the direction in which the curve $\eta \circ \varphi(\tau)$ crosses the image of the level set, $\eta\left(M_{s(\tau)}\right): \beta$ positive indicates an outward crossing, $\beta$ negative an inward one. Let $\varepsilon>0$ be small enough that $\beta(\tau)$ is negative along this trajectory for $\tau_{1}-\varepsilon \leq \tau \leq \tau_{1}$. The projected trajectory $\eta \circ \varphi(\tau)$ approaches $q$ as $\tau$ approaches $\tau_{1}$. Indeed, if $s_{1}$ is the level of $r$, we have that $\eta\left(M_{s(\tau)}\right)$ approaches $\eta\left(M_{s_{1}}\right)$ as $\tau$ approaches $\tau_{1}$. If $\eta \circ \varphi(\tau)$ approached $q$ from the exterior of $\eta\left(M_{s_{1}}\right)$, then $q$ would be an interior point of $A_{s_{1}}$, and consequently of $A_{s}$, which is impossible. Thus we have $\eta \circ \varphi(\tau)$ approaching $q$ from within the convex body bounded by $\eta\left(M_{s_{1}}\right)$. But $(\eta \circ \varphi)^{\prime}(\tau)$ is a multiple of the inward pointing normal to $\eta\left(M_{s(\tau)}\right)$, so $\eta\left(M_{s(\tau)}\right)$ does not converge to $\eta\left(M_{s_{1}}\right)$, which is also impossible. Therefore $\beta\left(\tau_{1}\right) \geq 0$.

Using this inequality yields the result that the boundary of $A_{s}$ is uniformly bounded for all $s$ in $\left(s_{0}, s^{*}\right)$. As above, let $\varphi(\tau):\left[\tau_{0}, \tau_{1}\right] \rightarrow M$ be an arclength parametrized trajectory of $\operatorname{grad} h$ with $\varphi\left(\tau_{0}\right)$ in $M_{s_{0}}$ and with $\eta \circ \varphi\left(\tau_{1}\right)$ on the boundary of $A_{s}$. The length of the projected trajectory, $L[\eta \circ \varphi]$, is bounded:

$$
L[\eta \circ \varphi]<\int_{\tau_{0}}^{\tau_{1}}\left[\left(1+s^{*}\right) /(1+s(t))\right] \beta(t) d t .
$$

[The factor $\left(1+s^{*}\right) /(1+s(t))$ is necessary to compensate for the fact that $\pi$ is not an isometry from $P_{s}$ to $P_{s^{*}}$.] Thus $L[\eta \circ \varphi]$ is no larger than $\left(s^{*} / s_{0}\right) \int_{\tau_{0}}^{\tau_{1}} \beta(t) d t$. Since $\beta\left(\tau_{1}\right) \geq 0$ and $\beta^{\prime}(\tau)<0$, then $\beta(\tau) \geq 0$ on the 
interval $\left[\tau_{0}, \tau_{1}\right]$. In addition, $\alpha(\tau)>0$ by the definition of $\operatorname{grad} h$, so we also have $\alpha^{\prime}(\tau) \geq 0$. Since $\alpha$ is positive on $M_{s_{0}}$, its minimum is positive, say $\alpha_{0}$. Then we have $\alpha(\tau) \geq \alpha_{0}$ since $\alpha^{\prime}(\tau)$ is nonnegative. Then:

$$
h\left(\varphi\left(\tau_{1}\right)\right)-h\left(\varphi\left(\tau_{0}\right)\right)=\int_{\tau_{0}}^{\tau_{1}} \alpha(t) d t \geq \alpha_{0}\left(\tau_{1}-\tau_{0}\right)
$$

Therefore $\tau_{1}-\tau_{0} \leq\left(s^{*}-s_{0}\right) / \alpha_{0}$. Since $\alpha(\tau) \geq \alpha_{0}$, then $\beta(\tau) \leq \sqrt{1-\alpha_{0}^{2}}$ so:

$$
\int_{\tau_{0}}^{\tau_{1}} \beta(t) d t \leq \int_{\tau_{0}}^{\tau_{1}} \sqrt{1-\alpha_{0}^{2}} d t \leq\left(\left(s^{*}-s_{0}\right) / \alpha_{0}\right) \sqrt{1-\alpha_{0}^{2}} .
$$

Hence $L[\eta \circ \varphi] \leq\left(s^{*} / s_{0}\right)\left(\left(s^{*}-s_{0}\right) / \alpha_{0}\right) \sqrt{1-\alpha_{0}^{2}}$ and $L[\eta \circ \varphi]$ is uniformly bounded. Since the boundary of $A_{s^{*}}$ is the limit of boundaries of $A_{s}$ as $s$ approaches $s^{*}$, this calculation shows that the boundary of $A_{s^{*}}$ is bounded, so $A_{s^{*}}$ is bounded as well. But this implies that $\eta\left(M^{*}\right)$, which is $M^{*}$, is bounded. Thus, if case (iii) were possible, $M^{*}$ would be complete, bounded, and noncompact, which is clearly impossible.

Only two cases remain. In case (ii), in which $D^{*} \neq M$ and $M^{*}$ contains a critical point of $h$, the conclusions of the theorem follow. Indeed, let $q$ denote a critical point of $h$ in $M^{*}$. Since $q$ is a critical point of $h$, then $\nu_{q}= \pm X_{0}$. If $\nu_{q}$ were $-X_{0}$, then Lemma 1 would imply that $q$ has a neighborhood on which $h \geq s^{*}$. But then $q$ would not be in $\mathrm{Cl}\left[D^{*}\right]$, since $h<s^{*}$ on $D^{*}$. Consequently, $\nu_{q}=+X_{0}$. A calculation like the one of $\S 2$ shows that the Hessian of $h$ at $q$ is negative definite: $(1+h(q)) \nabla^{2} h(q)=-$ II $_{q}-$ Id . Therefore $q$ is a nondegenerate maximum of $h$; and so there is a neighborhood of $q$ which is entirely contained in levels $P_{s}$ for $s \leq s^{*}$ and which has $q$ as its only point in $P_{s^{*}}$. In other words, $M^{*}=\{q\}$. Since each of the levels $M_{s}$ is imbedded in $P_{s}$, then $M$ is imbedded in $H^{n+1}$. Since II is positive definite and $M$ is imbedded, then $M$ bounds a convex body (see van Heijenoort [11]). Since $M$ bounds a convex body and $h$ is a Morse function with exactly two critical points, then $M$ is diffeomorphic to $S^{n}$.

To complete the proof of Theorem A, it is only necessary to show that case (i), $D^{*}=M$, is impossible. Note that in either case (i) or case (ii), the horosphere $P_{0}$ globally supports $f(M)$. The condition that the eigenvalues of II are strictly greater than 1 is an open condition; it holds in a neighborhood of $p$. On this neighborhood, no other point has normal $-X_{0}$, which is $\nu_{p}$. Repeating the entire argument from a second point $p^{\prime}$ in this neighborhood shows that $f(M)$ is contained within the closed horoball tangent to $f(M)$ at $p^{\prime}$. Since $\nu_{p^{\prime}} \neq \nu_{p}$, these two horoballs have different points at infinity; therefore their intersection is compact. Since $M$ is a complete, imbedded subset of the intersection, $M$ must be compact and $h$ must have a maximum, which is a critical point of $h$ other than $p$. Therefore $D^{*} \neq M$, and case (i) cannot hold. This completes the proof of Theorem A. 
The situation in Euclidean space as treated by do Carmo and Lima is closely analogous to Theorem $\mathrm{A}$. It is assumed that $M$ is a complete, connected, $C^{\infty}$ Riemannian manifold whose dimension, $n$, is at least 2 ; that $f: M \rightarrow \mathbf{R}^{n+1}$ is a $C^{\infty}$ isometric immersion; that there exists a smooth normal field $\nu$ along $f$ such that, at every point of $M$, the second fundamental form is semidefinite; that there exists a point $p$ at which $\mathrm{II}_{p}$ is positive definite. It is concluded, in part, that $M$ is diffeomorphic to $S^{n}$ or $\mathbf{R}^{n}$ and is imbedded as the boundary of a convex body. The proof is structured similarly to the above proof of Theroem A: consider a height function $h$, the distance above $T_{p} M$. Using this height function, define normal values and normal regions, arriving at a largest such region $D^{*}$. Consider the three cases: (i) $D^{*}=M$; (ii) $D^{*} \neq M$ and the boundary of $D^{*}$, denoted $M^{*}$, contains a critical point of $h$; or (iii) $D^{*} \neq M$ and $M^{*}$ contains no critical point of $h$. To prove the impossibility of case (iii), it is shown that $M^{*}$ is a complete, noncompact hypersurface of the hyperplane of $\mathbf{R}^{n+1}$ at height $s^{*}$ that is the boundary of a convex body. The desired contradition is that the total curvature of $M^{*}$ must be less than or equal to $\pi$, since $M^{*}$ is a noncompact, convex hypersurface of $P_{s^{*}}$, while the total curvature of the compact hypersurfaces at lower levels is $2 \pi$, and the total curvature, being an integral, is a continuous function of the height parameter.

This last assertion need not always hold, since although the integrand is continuous, the measure of the image of the Gauss map may change discontinuously. One example is the hypersurface of $\mathbf{R}^{4}$ defined by the equation $x_{4}=\left(x_{1}^{2}+x_{2}^{2}+x_{3}^{2}\right) /\left(x_{1}^{2}+x_{2}^{2}+1\right)$. The height function $h$, or $x_{4}$, has a nondegenerate minimum at the origin. The level surfaces $x_{4}=c$ for $0<c<1$ are ellipsoids, whose total curvature is $2 \pi$. But for $c=1$, the level surface becomes $\left\{x_{3}= \pm 1\right\}$, the union of two planes, whose total curvature is zero. This example does not falsify do Carmo and Lima's result, however, since the second fundamental form is indefinite at $\left(x_{1}, x_{2}, x_{3}\right)$ if $x_{1}^{2}+x_{2}^{2}>1$.

One way to demonstrate a contradiction would be to show, as in the previous proof, that $M^{*}$ is bounded. In the case that $n$ is greater than 2 , this can be done as follows. Fix coordinates on $\mathbf{R}^{n+1}$ of the form $\left(x_{0}, x_{1}, \ldots, x_{n}\right)$ with $p$ as the origin and $\nu_{p}=-X_{0}$, so that the coordinate function $x_{0}$ is the height function $h$. Fix a small positive $\varepsilon$ for which the normal level surface $M_{\varepsilon}$ exists, and on which II is positive definite everywhere. The normal level surfaces between $M_{\varepsilon}$ and $M^{*}$ contain no critical points of $h$, so choose the normal $\nu_{s}$ to the level surface $M_{s}$ in the hyperplane $\left\{x_{0}=s\right\}$ which satisfies the inequality $\left\langle\nu, \nu_{s}\right\rangle>0$. For each $s$ in the interval $\left[\varepsilon, s^{*}\right)$, the level surface $M_{s}$ is compact, is of dimension at least 2 , and has semidefinite second fundamental form in $P_{s}$ with respect to $\nu_{s}$. Therefore, according to Proposition 1 of do Carmo and Lima [4], $M_{s}$ is imbedded as the boundary of a convex body. The choice of normal $\nu_{\varepsilon}$ given by $\left\langle\nu, \nu_{\varepsilon}\right\rangle>0$ is the outward normal, so the normal $\nu_{s}$ is the outward normal for all $s$ in $\left[\varepsilon, s^{*}\right)$. Further, since $M_{s}$ is convex, II $_{s}(U, U) \geq 0$ for all vector fields $U$ tangent to $M_{s}$. 
The region between $M_{\varepsilon}$ and $M^{*}$ is covered by trajectories of $\operatorname{grad} h$, each of which contains a point of $M_{\varepsilon}$. Parametrize these trajectories as $\varphi(t)$ by arclength from their points in $M_{\varepsilon}$. We shall see that $\operatorname{II}\left(\varphi^{\prime}, \varphi^{\prime}\right) \geq 0$. Suppose, to the contrary, that the inequality $\operatorname{II}\left(\varphi^{\prime}, \varphi^{\prime}\right)<0$ holds at some $q$ between $M_{\varepsilon}$ and $M^{*}$. Let $V$ be the maximal connected, open set containing $q$ on which $\operatorname{II}\left(\varphi^{\prime}, \varphi^{\prime}\right)<0$. Let $U$ be a vector field on $V$ which is tangent to the slices $M_{s}$.

Since $\operatorname{II} s(U, U) \geq 0$ and $\left\langle\nu, \nu_{s}\right\rangle>0$, then $\operatorname{II}(U, U) \geq 0$. But II is semidefinite, so $\operatorname{II}(U, U)=0$ and $\operatorname{II}\left(U, \varphi^{\prime}\right)=0$. For any $s$ in $\left[\varepsilon, s^{*}\right)$, we find that the portion of $M_{s}$ inside $V$ is flat and that the vector field $\varphi^{\prime}$ is parallel along $M_{s} \cap V$. Then, using the Codazzi-Mainardi equation, we find that $\nabla_{U}\left(\mathrm{II}\left(\varphi^{\prime}, \varphi^{\prime}\right)\right)=0$.

Since $\operatorname{II}\left(\varphi^{\prime}, \varphi^{\prime}\right)$ is constant on $M_{s} \cap V$ and since $M$ is complete, then we have $M_{s} \cap V$ is a hyperplane in $P_{s}$. But this contradicts the fact that $M_{s}$ is compact; hence $\operatorname{II}\left(\varphi^{\prime}, \varphi^{\prime}\right) \geq 0$ everywhere. A calculation similar to the one in Theroem A shows that the length of the projection onto $P_{s^{*}}$ of the trajectory $\varphi(t)$ satisfies the inequality $L[\eta \circ \varphi] \leq\left(\left(s^{*}-\varepsilon\right) / \alpha\right) \sqrt{1-\alpha^{2}}$, where $\alpha$ is the minimum value of $\left\langle\nu, \nu_{\varepsilon}\right\rangle$ on $M_{\varepsilon}$. This completes the proof that $M^{*}$ is bounded.

This proof depends on the fact that the levels $M_{s}$ are convex, which follows if the dimension of $M$ exceeds 2 . Perhaps a special argument would establish the case that $\operatorname{dim} M=2$ without resorting to Sacksteder's more complicated techniques.

\section{THE PROOF OF THEOREM B}

This section will be concerned with the proof of

Theorem B. Suppose $M$ is a complete, connected $C^{\infty}$ Riemannian manifold whose dimension, $n$, is at least 2. Suppose that $f: M \rightarrow H^{n+1}$ is a $C^{\infty}$ isometric immersion and that there exists a smooth normal field $\nu$ along $f$ such that, at every point of $M$, all the eigenvalues of the second fundamental form of $M$ in $H^{n+1}$ with respect to $\nu$ are at least one. If the least eigenvalue of II at every point is 1 , then (1) $M$ is isometric to $R^{n}$, and (2) $M$ is imbedded in $H^{n+1}$ as a horosphere.

Proof. Define a distribution $E$ by $E_{p}=\left\{X_{p}\right.$ in $\left.T_{p} M: \nabla_{X_{p}} \nu=X_{p}\right\}$, that is, the distribution of eigenvectors of II having eigenvalue 1 ; an integer $k$ as $\min \left\{\operatorname{dim} E_{p}: p\right.$ in $\left.M\right\} ;$ an open set $U$ as $\left\{p\right.$ in $\left.M: \operatorname{dim} E_{p}=k\right\}$. By hypothesis, $k$ is at least 1 . In terms of these definitions, a theorem of Reckziegel [9] specializes to give the results that (1) the distribution $E$, on $U$, is integrable; (2) each leaf $L$ of the foliation $\mathscr{F}$ obtained by integrating $E$ on $U$ is a $k$-dimensional umbilical submanifold of $M$, and $f \mid L: L \rightarrow H^{n+1}$ is an umbilical immersion; and (3) all the leaves of $\mathscr{F}$ are complete.

Fix a point $p$ in $U$ and let $L$ be the leaf of $\mathscr{F}$ through $p$. Since $\nu$, the normal to $M$, is also the mean curvature vector of $L$ along $f \mid L$, then the 
distribution spanned by $E$ and $\nu$ is parallel along $L$. Therefore, the image of $L$ is an open set in some $k$-dimensional horosphere in some $H^{k+1}$ contained in $H^{n+1}$. Since $L$ is complete, $L$ is the entire $k$-dimensional horosphere.

Define a $k$-flat in $H^{n+1}$ to be a $k$-dimensional linear subspace of a horosphere. Say that two $k$-flats are parallel if they are contained in concentric horospheres and are parallel in the upper half-space coordinates for which the horospheres are described by equations $x_{0}=$ constant.

We shall see that $f(M)$ is foliated by $k$-flats that are parallel to $L$. From this fact we shall deduce that $f(M)$ is contained in a single horosphere. Then, since $M$ is complete, $f(M)$ is the entire horosphere.

Fix coordinates on $H^{n+1}$ for which $p=(1,0, \ldots, 0)$, for which $\nu \mid L=$ $-X_{0}$, and for which $f(L)$ is the $k$-flat in the $\left\{x_{1}, \ldots, x_{k}\right\}$ coordinate directions. Let $C$ denote the set of points in $M$ which belong to preimages of $k$-flats in $f(M)$ parallel to $f(L)$. Then $C$ is nonempty, since $C$ contains $L$; and $C$ is closed, since parallel $k$-planes in $\mathbf{R}^{n+1}$ converge uniformly and $M$ is complete.

Let $C_{a}$ be a component of $C$ in $M$. Since $X_{1}$ is tangent to $C_{a}$ everywhere, and since $\nabla_{X_{1}} X_{1}=X_{0}$, then at every point of $C_{a}, \mathrm{II}\left(X_{1}, X_{1}\right)=-\left\langle X_{0}, \nu\right\rangle$. The hypothesis on the eigenvalues of II implies that $\mathrm{II}(Z, Z) \geq 1$ for every unit tangent vector $Z$. In this case, this is possible only if $\nu=-X_{0}$ for every point of $C_{a}$. Every point of $C_{a}$ is contained in a $k$-flat parallel to $L$, so $\partial h / \partial x_{i}=0$ for $i=1, \ldots, k$ where $h$ is the pull-back along $f$ of the coordinate function $x_{0}$. The result on the normal implies that, in addition, $\partial h / \partial x_{i}=0$ for $i=k+1, \ldots, n$. By a theorem of Morse [8], $h$ is constant on $C_{a}$, that is, $f\left(C_{a}\right)$ is contained in a horosphere.

Since $M$ is connected and $C$ is closed, it only remains to prove that $C$ is open, in which case $C$ will have only one component, namely all of $M$, and $f(M)$ will be contained in a horosphere. Then, since $M$ is complete, we shall have that $f$ maps $M$ isometrically only the entire horosphere.

It suffices to show that $C$ contains a neighborhood of $p$. For notational convenience, assume that $k=1$ and $n=2$; there is no difficulty in writing out the higher dimensional cases. Define $W$ as the component of the set $\{q$ in $M$ : $\left.\left\langle\nu_{q}, X_{0}\right\rangle<0\right\}$ that contains $L$. Let $P$ be the horosphere $\left\{x_{0}=1\right\}$, which contains $f(L)$, let $\pi: H^{3} \rightarrow P$ be the projection along vertical geodesics tangent to $X_{0}$, and let $\eta: M \rightarrow P$ denote the composition $\pi \circ f$. Note that, on $W, \eta$ is a local diffeomorphism.

Since $\nu_{p}=-X_{0}$, then $p$ has a neighborhood $V$ on which $\eta$ is a diffeomorphism. Choose $\varepsilon$ small enough that the point $(1,0, \varepsilon)$ is contained in $\eta(V)$, and denote its pre-image in $V$ by $q$. Let $T_{t}$ be the solid triangle in $P$ whose vertices are $(1,0,0),(1,0, \varepsilon)$, and $(1, t, 0)$, and let $\Delta_{t}$ be the component of $\eta^{-1}\left(T_{t}\right)$ that contains $p$. Let $s$ be the supremum of all $t$ for which $\eta \mid \Delta_{t}$ is a diffeomorphism onto $T_{t}$. Certainly $s$ is positive, since $\eta$ is a diffeomorphism 
on any set contained within $V$. Let $\Delta^{*}=\bigcup_{0<t<s} \Delta_{t}$; then $\eta$ is a diffeomorphism on $\Delta^{*}$, since $\Delta_{t}$ is a subset of $\Delta_{\tau}$ if $0<t<\tau<s$. [The higher dimensional version uses for $T_{r}$ the convex hull in $P$ of $\eta(q)$ and the ball $B_{r}^{k}(0)$, of dimension $k$, of radius $r$, centered at $\eta(p)$, contained in $f(L)$.]

We shall see that $s=+\infty$. Suppose, on the contrary, that $s$ is finite. First, we shall derive that $f\left(\Delta^{*}\right)$ is bounded. Over the set $\eta\left(\Delta^{*}\right)$, the set $f\left(\Delta^{*}\right)$ can be represented as the graph of the function $h$. The proof of Lemma 1 shows that this function is convex. On $L$, which includes the edge of $\Delta^{*}$ opposite from $q, h=1$ and $\nu=-X_{0}$. The latter implies that $\left(\partial h / \partial x_{i}\right) \mid L=0$ for $i=1,2$. Together with the convexity of $h$, this implies that, for each $r$ in $\Delta^{*}, 1 \leq h(r) \leq h(q)$. Therefore the distance between two points $u_{1}$ and $u_{2}$ of $f\left(\Delta^{*}\right)$ satisfies:

$$
\begin{aligned}
d\left(u_{1}, u_{2}\right) & \leq d\left(u_{1}, \pi\left(u_{1}\right)\right)+d\left(\pi\left(u_{1}\right), \pi\left(u_{2}\right)\right)+d\left(\pi\left(u_{2}\right), u_{2}\right) \\
& \leq \log (h(q))+d\left(\pi\left(u_{1}\right), \pi\left(u_{2}\right)\right)+\log (h(q)) .
\end{aligned}
$$

The distance from $\pi\left(u_{1}\right)$ to $\pi\left(u_{2}\right)$ is bounded by the distance from $\eta(q)$ to $(1, s, 0)$, hence $f\left(\Delta^{*}\right)$ is bounded. This implies that $f\left(\mathrm{Cl}\left[\Delta^{*}\right]\right)$ can be described as the graph of $h$ over $T_{s}$.

Next we show that $\mathrm{Cl}\left[\Delta^{*}\right]$ is contained in $W$. Every point of $\Delta^{*}$ is contained in $W$, so if there is a point $r$ in $\mathrm{Cl}\left[\Delta^{*}\right]$ that is not in $W$, then $r$ is in $\partial W$ and $\left\langle\nu_{r}, X_{0}\right\rangle=0$. This can only happen for points whose image $\eta(r)$ lies in the interior of the segment in $P$ joining $\eta(q)$ to $(1, s, 0)$. Therefore $\nu_{r}$ is perpendicular to the direction from $\eta(q)$ to $(1, s, 0)$; but the Lemma of $\S 2$, which states that $f(M)$ is locally supported by its tangent horosphere at $r$, implies that $f\left(\Delta^{*}\right)$ is not the graph of a function over all of $\eta\left(\Delta^{*}\right)$, which is impossible. Therefore, there is not such $r$ and $\mathrm{Cl}\left[\Delta^{*}\right]$ is contained in $W$.

We have just seen that, at every point of $\mathrm{Cl}\left[\Delta^{*}\right]$, the normal satisfies $\left\langle\nu, X_{0}\right\rangle$ $<0$, so the projection $\pi \mid f\left(\mathrm{Cl}\left[\Delta^{*}\right]\right)$ is a diffeomorphism onto $T_{s}$. This implies that $\eta \mid \mathrm{Cl}\left[\Delta^{*}\right]$ is a diffeomorphism onto $T_{s}$. Now we may write $\Delta_{s}$ for $\mathrm{Cl}\left[\Delta^{*}\right]$. But $\Delta_{s}$ is compact and contained in $W$, so there exist larger triangles $\Delta_{s^{\prime}}$, and $T_{s^{\prime}}$ with $\eta \mid \Delta_{s^{\prime}}$ a diffeomorphism onto $T_{s^{\prime}}$. This contradicts the maximality of $s$, so no such finite $s$ exists; that is, $s=+\infty$.

Similarly, the infimum of $\left\{t<0: \eta \mid \Delta_{t}\right.$ is a diffeomorphism onto $\left.T_{t}\right\}$ is $-\infty$. Thus a portion of $f(M)$ can be represented as the graph of the convex function $h$ over the strip in $P$ where $-\infty<x_{1}<+\infty$ and $0 \leq x_{2}<\varepsilon$. If there were a point $\left(y_{1}, y_{2}\right)$ whee $\partial h / \partial x_{1} \neq 0$, then the convexity of $h$ would imply:

$$
1=h\left(y_{1}+t, 0\right) \geq h\left(y_{1}, y_{2}\right)+t \frac{\partial h}{\partial x_{1}}\left(y_{1}, y_{2}\right)-y_{2} \frac{\partial h}{\partial x_{2}}\left(y_{1}, y_{2}\right) .
$$

Taking $|t|$ sufficiently large makes the expression $t\left(\partial h / \partial x_{1}\right)\left(y_{1}, y_{2}\right)$ exceed any bound. Since $\partial h / \partial x_{1}=0$, the function $h$ is constant over lines in the strip in the $x_{1}$-direction. It follows that $C$ is open; and this completes the proof of Theorem $B$. 


\section{REFERENCES}

1. S. B. Alexander, Locally convex hypersurfaces of negatively curved spaces, Proc. Amer. Math. Soc. 64 (1977), 321-325.

2. J. Bolton, Isometric immersions into manifolds without conjugate points, Math. Proc. Cambridge Philos. Soc. 92 (1982), 243-250.

3. J. de Miranda Gomes, On isometric immersions with semi-definite second quadratic forms, An. Acad. Brasil. Cienc. 55 (1983), 145-146.

4. M. do Carmo and E. Lima, Immersions of manifolds with non-negative sectional curvatures, Bol. Soc. Brasil. Mat. 2 (1971), 9-22.

5. M. do Carmo and F. Warner, Rigidity and convexity of hypersurfaces in spheres, J. Differential Geometry 4 (1970), 133-144.

6. J. Hadamard, Sur certaines propriétés des trajectories en dynamique J. Math. Pures Appl. 3 (1897), 331-387.

7. L. B. Jonker, Immersions with semi-definite second fundamental forms, Canad. J. Math. 27 (1975), 610-617.

8. A. P. Morse, The behavior of a function on its critical set, Ann. of Math. 40 (1939), 62-70.

9. H. Reckziegel, Completeness of curvature surfaces of an isometric immersion, J. Differential Geometry 14 (1979), 7-20.

10. R. Sacksteder, On hypersurfaces with no negative sectional curvatures, Amer. J. Math. 82 (1960), 609-630.

11. J. van Heijenoort, On locally convex manifolds, Comm. Pure Appl. Math. 5 (1952), 223-242.

Department of Mathematics, Smith College, Northampton, Massachusetts 01063 